Original Research Paper

\title{
Porosity Analysis in Porous Brass Using Dual Approaches
}

\author{
${ }^{1}$ Ryan Yeargin, ${ }^{2}$ Rob Ramey and ${ }^{2}$ Cindy Waters \\ ${ }^{I}$ Department of Civil, Architectural and Environmental Engineering, \\ ${ }^{2}$ Department of Mechanical Engineering, \\ North Carolina A\&T State University, Greensboro, NC, USA
}

Article history

Received: 15-02-2016

Revised: 17-02-2016

Accepted: 20-02-2016

Corresponding Author:

Cindy Waters

Department of Mechanical

Engineering, North Carolina A\&T

State University, Greensboro, NC, USA

Email:kwaters@ncat.edu

\begin{abstract}
The goal of this research is to produce porous samples for various applications such as heat exchangers, filters and radiation shields. The level of porosity is an important objective yet the level of confidence in two main methods (hand calculations and microscopic analysis) is not high. This work will compare porosity values obtained from traditional hand calculations against analysis from microscopic imaging. Brass powder was mixed with sodium chloride $(\mathrm{NaCl})$ and Polyvinyl Alcohol (PVA) to create porous brass to examine the different levels of porosity in the porous brass. Porous brass was fabricated by powder metallurgy using the dissolution sintering technique by mixing brass with $\mathrm{NaCl}$ to obtain the volume ratios of $1: 0.5,1: 1,1: 1.5$ and $1: 2$ brass to sodium chloride. The expected porosities are as follows: 1:0.5-33, 1:1-50, 1:1.5-60 and 1:2$67 \%$. In order to create a mixture of the desired volume ratios the respective density of each component must be factored into an equation to determine the measured mass for mixing. The $\mathrm{NaCl}$ was leached away for 2 hours in $90^{\circ} \mathrm{C}$ water after sintering. Scanning Electron Microscopy (SEM) was used to confirm the morphology of the porous brass. It was also used to observe the particle size while viewing the particle's shape and surface appearance. SEM imaging showed a hierarchical bimodal structure existing of macropores and micropores throughout all samples. The porous samples were polished and their micro pore morphology was captured at three different levels.
\end{abstract}

Keywords: Brass, $\mathrm{NaCl}$, Porous, Sintering, Leaching

\section{Introduction}

This paper discusses the fabrication of porous samples using the powder metallurgy method. It also examines porosity values taken from powder metallurgical formulas and electronic microscopic software. Porous samples or metal foams are used as heat exchangers, biomedical implants, filters, etc. The powder metallurgy method was chosen based on the relatively low cost, control factor and chemical reactivity of brass with atmospheric gases and mold materials during experimentation. The porosity in each sample is dependent upon the metal to space holder ratio. In this study 4 different relative volume ratios were evaluated and analyzed by their mechanical properties. The volume ratios were $1: 0.5,1: 1,1: 1.5,1: 2$ metal to space holder respectively. Volume ratios determine how much space holder material and metal should be mixed to obtain the targeted mass. SEM imaging was used to examine each porous sample in great detail.

\section{Background}

Powder Metallurgy (PM) is a rising technology especially now with the explosion of 3D metal printing. The European Market alone has an annual turnover of over six billion Euros, with annual worldwide metal powder production exceeding one million tons (EMPA, 2015). Now there is an interest in creating porous metals via this method. In the biomedical realm there is a need for porous metals for bone in growth and scaffold fabrication with the space holder method relies on temporary particles added to metallic matrix powder, i.e., space holding particles that act as a pore former (Arifvianto and Zhou, 2014). 
PM is a manufacturing process where various powder metals such as stainless steel, brass, copper, iron and bronze are compressed and then sintered (heated) to increase adhesion between particles. Blended material allow for a certain amount of customization of material properties to produce desired characteristics suitable for a particular application. Compressing the powder metal, or blending metal powders forms the part. The compressed pieces are then sintered in an atmosphere-controlled furnace at high temperatures, causing the metal powder particles to be metallurgically bonded together. This remarkably "green" and environmentally friendly process uses $99 \%$ of the material that's in the mold, producing harmless bi-products of nitrogen and hydrogen (ASI, 2015). Powder metallurgy also allows manufactures to make more products that are consistent and predictable in application. Manufactures can tailor the physical characteristics of the product to meet the performance requirements of the consumer. By using powder metallurgy engineers are able to control the porosity, performance, precision, stress, absorbing vibrations, hardness, wear resistance and surface finish.

Controlling the porosity and density is very important when fabricating porous metals. Porosity is a ratio expressed as a percentage of the volume of the pores or interstices of a substance, as a rock or rock stratum to the total volume of the mass (Dictionary.com, 2015). The mass of atoms, their size and how they are arranged determine the density of a substance. Therefore objects with the same volume but different mass have different densities.

A major classification of porous metals, or metal foams, is between open-cell and closed-cell. These are formed by a method that uses space-holding particles which are mixed and compacted together with metallic matrix powder particles and then removed either before or during sintering, leaving new pores behind in the matrix. The space holder method may also be called the fugitive filler method (Hong et al., 2008). In closed-cell foams each cell is completely enclosed by a thin wall or membrane of metal, whilst in open-cell foams the individual cells are interconnected (Matassi et al., 2013). Porous and foamed metals exhibit various characteristics that differ from bulk metals, including possessing a low density and large surface area. These metals are expected to be used as lightweight materials, catalyst carriers, electrodes, vibration and acoustic energy damping materials, impact energy absorption materials, etc. However, porous and foamed metals all suffer from deteriorating mechanical properties such as strength, stiffness and fatigue due to the inhomogeneous pore number density distribution and pore size (Nakajima, 2010).
Various techniques have been used to produce porous metals and PM method is one of them. The PM method involves the use of a space holder and examples of the spacers include magnesium, $\mathrm{NaCl}$ and carbamide (urea) etc. The space holder can be later removed by leaching out in hot water or by thermal removal (Sharma et al., 2011; Tuncer and Arslan, 2009). The choice of $\mathrm{NaCl}$ as space holder in this study was due to its semi-spherical shape, which creates more uniform macro pores. If there is an increase in porosities with an acicular pore shapes there is frequently a corresponding decrease in the strength of the foam (Esen and Bor, 2011).

\section{Experimental Plan}

\section{Sample Preparation}

Less than 325 microns of brass powder and sodium chloride $(\mathrm{NaCl})$, ranging from 50-100 microns, were used to obtain the following sample ratios 1:0.5, 1:1, $1: 1.5$ and $1: 2$. Composition calculations are shown below in Fig. 1. Four $1 / 2$ in samples of each volume ratio were prepared; each sample had a target mass of $3 \mathrm{~g}$. Two drops of Polyvinyl Alcohol (PVA) were added to each composition and mixed with a mortar and pestle for five minutes for complete homogenization. The sample was then poured into a $1 / 2$ " diameter press die and then compacted with a uniaxial load of $375 \mathrm{MPa}$. The samples were then set out to dry at room temperature for $1 \mathrm{~h}$. Once dried the samples were then sintered for $81 / 2 \mathrm{~h}$. The sintering process involved placing the samples in a GSL-1700X high temperature vertical tube furnace which uses MoSi2 as heating elements with 4" diameter high purity alumina tube all purchased from MTI. The temperature was controlled by high precision controller with the accuracy $+/-1^{\circ} \mathrm{C}$. The chamber of the furnace was flushed with argon gas and purged twice to remove all other gases present in the chamber after which vacuum was achieved using a pump with maximum vacuum pressure. The rate of sintering is dictated by both the driving force and the mobility of the atoms, which depends on the sintering temperature. The activation energy for sintering scales with the melting temperature of the metal $(\mathrm{Q}(\mathrm{kJ} / \mathrm{mol})=0.145 \mathrm{Tmp})$. The furnace was heated at the rate of $10^{\circ} \mathrm{C} / \mathrm{min}$ up to $200^{\circ} \mathrm{C}$ in order to evaporate the water and PVA completely. The furnace was then heated to $850^{\circ} \mathrm{C}$ at $5^{\circ} \mathrm{C} / \mathrm{min}$ increments. This temperature was held for 4 hours then cooled to room temperature $\left(20^{\circ} \mathrm{C}\right)$ at the rate of $10^{\circ} \mathrm{C} / \mathrm{min}$. After sintering, the samples were leached for $2 \mathrm{~h}$. The dissolution process or leaching dissolves the $\mathrm{NaCl}$ in the sample. Samples were placed in a tea sieve and immersed in water at $90^{\circ} \mathrm{C}$. The tea sieve was suspended in the beaker with a small magnetic stirrer at the bottom. The beaker was heated on a hot plate to control the temperature of the water. 


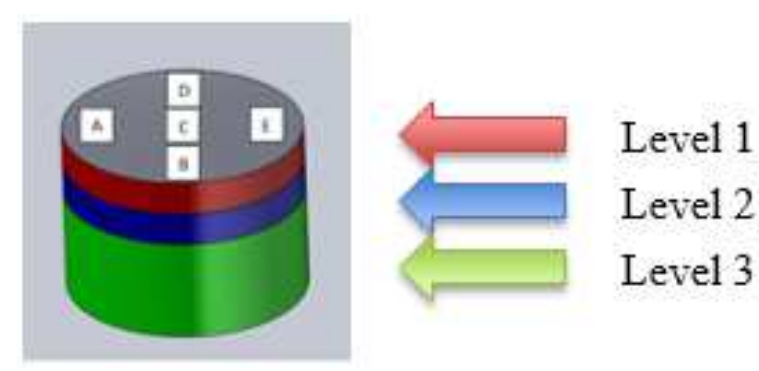

Fig. 1. Schematic of whole sample, A-E represents locations where micrographs were taken at each of the three levels

The final stage of dissolution occurred when the samples were placed below running water for $15 \mathrm{~min}$ to ensure that the remaining space holder was removed and the sample was dried in a muffle furnace to the temperature of $120^{\circ} \mathrm{C}$ for $2 \mathrm{~h}$ (Esen and Bor, 2011):

$$
\begin{aligned}
& M_{A}=\frac{M_{T}}{\frac{\rho_{B}}{\rho_{A}}\left(\frac{1}{\vartheta_{A}}-1\right)+1} \\
& M_{A}+M_{B}=M_{T}
\end{aligned}
$$

Where:

$$
\begin{aligned}
M_{A}= & \text { Mass of metal in grams } \\
M_{B}= & \text { Mass of space holder in grams } \\
M_{T}= & \text { Total mass of the metal and the space holder in } \\
& \text { grams } \\
\rho_{A}= & \text { Density of the metal in } \mathrm{g} / \mathrm{cm}^{3} \\
\rho_{B}= & \text { Density of the space holder in } \mathrm{g} / \mathrm{cm}^{3} \\
\vartheta_{A}= & \text { Volume fraction of the metal }
\end{aligned}
$$

\section{Hardening, Grinding and Polishing}

Before the brass samples could be viewed they had to be encased in epoxy to give the porous structure stability. Then the samples were ground and polished in order to have a clear view of the pores in each sample and to ensure a flat level of the brass sample for optical light viewing. The sample was polished with 0.5alumina powder solution applied to a 10 in CERMESH metal mesh cloth. After the samples were polished they were then ready to be viewed under the microscope. A procedure was developed to image the sample at three levels from the uppermost surface to garner more varied porosity information.

\section{Microscopic Imaging}

A Leica DM1750 M microscope was used to observe all analyzed porous brass samples. The first level was the finished level of the ground and polished surface. A vernier caliper was used to measure the height and diameter of each sample. Levels 2 and 3 were chosen based off of the salt size, which was 100 microns. 100 microns was then increased by a factor of two, which would ensure that the next depth of image (level 2) would provide the most contrast possible in porous structure. After level 2 of the sample was viewed the grinding process was repeated to a new depth of $0.2 \mathrm{~mm}$ to obtain level 3 images. On each level 5 images were captured as shown A-E in Fig. 1.

\section{Results}

\section{Scanning Electron Microscopy Imaging}

Figure 2 illustrates the SEM images at 50, 500, 2000 and $5000 \mathrm{x}$ respectively. Sintering for volume ratio $1: 1$ was the least efficient process. The 1:1 sample had the most sodium chloride existing in the sample. Big white spots are visible on the surface of the 1:1 sample as seen in $50 \mathrm{x}$. At $500 \mathrm{x}$ the surface of sample 1:1 is covered in sodium chloride crystals. At $2 \mathrm{Kx}$ a better view of the crystals can now be seen on sample 1:1. The crystals look like white bumps all over the surface. At $4 \mathrm{Kx}$ the crystals do not look like dots or circles at this magnification. They are square and rectangular shaped as seen in sample 1:1.

\section{Hand Calculation Analysis:}

$$
\begin{aligned}
& V_{T}=\frac{\pi D^{2} H}{4} \quad \rho_{\text {porous }}=\frac{M_{T}}{V_{T}} \\
& V_{A}=\frac{1}{1+S_{H}} P=1-\left(\frac{M_{T}-M_{A}}{M_{A}}\right)\left(\frac{\rho_{\text {porous }} V_{A}}{\rho_{B}\left(1-V_{A}\right)}\right)
\end{aligned}
$$

Where:

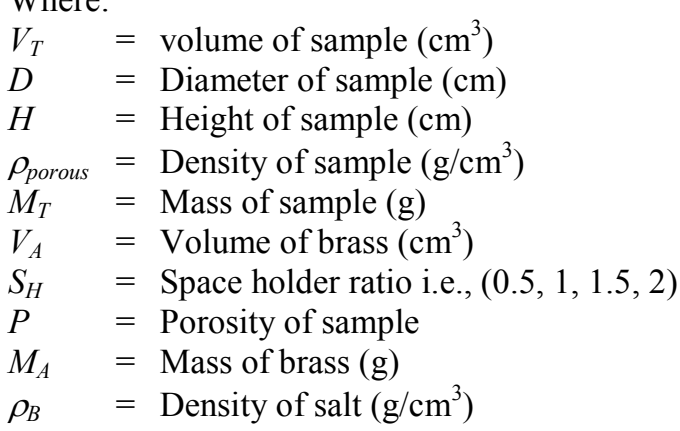

Hand calculations were also used to determine the porosity in each sample. The porosity in each sample was calculated using the Equation 1-4. The expected value was calculated using Equation 3. Equation 3 calculates the approximated volume of density, which can also be called the approximated density for each volume ratio. The expected porosity for each volume ratio is found by subtracting the approximated density from 1; For example the expected porosity for a volume 
ratio of 1:1 with an approximated density of $50 \%$ would be, $1-0.50=0.50 \times 100=50 \%$. Equation 1, 2 and 4 use parameters that are specifically tied to each individual sample, such as height, diameter and mass. Table 1 shows the average of all four samples for each ratio. The four sample averages are then averaged to determine the grand average. The grand average in comparison to the expected is very close for ratio 1:0.5 but as the ratio of salt becomes bigger the error between the grand average and expected becomes bigger as well.

\section{Microscopic Analysis}

Figure 3a was taken using a Stereo microscope with a total magnification of $10 \mathrm{x}$. Figure $3 \mathrm{~b}-\mathrm{d}$ were taken using the Leica DM1750 M microscope with a total magnification of $50 \mathrm{x}$. Each microscopic image was converted to black and white to set a standard for pore detection. The light area is brass and the dark area is the porous area, Fig. 3c. The pores were then color-coded according size, next the porosity of each image was calculated using the image analysis of the microscope software. Table 2 shows a non-uniform pore distribution between the levels. This is evidence that a homogeneous sample was not formed. The grand average values for each volume ratio differ significantly from the expected

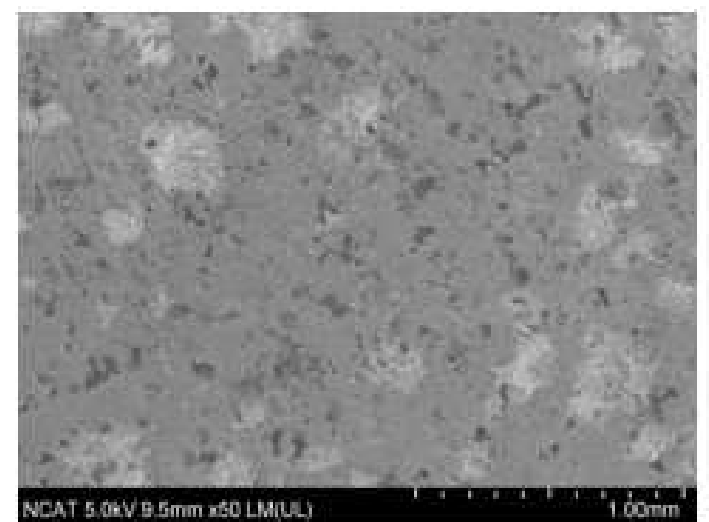

(a)

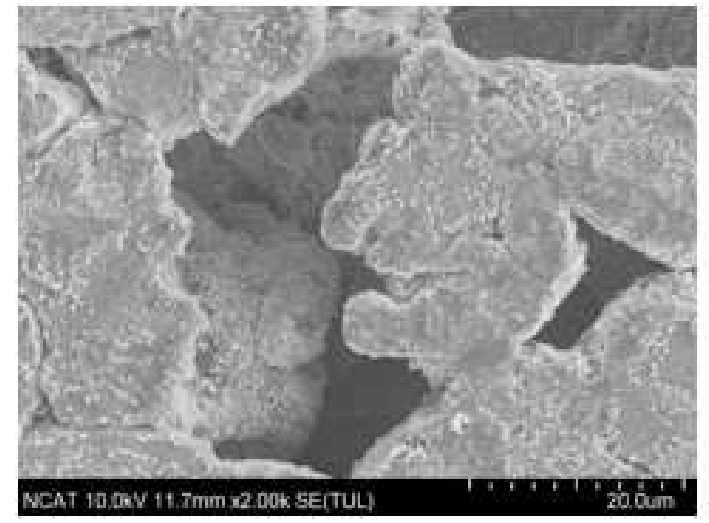

(c) value. The Percent difference of the calculated and microscopic values are tabulated in Table 3.

\section{Discussion}

Considering Table 3 ratio 1:0.5, there is a 6 to $21 \%$ difference in the calculated to microscopic values versus expected. In addition, for the ratio $1: 1$ there is a 6 to $14 \%$ difference. These are very high values of error with the microscopic imaging having the most error. The difference in calculated versus expected values originates from the mixing and pressing of the sample. During mixing and pressing some of the brass and or salt is lost in the shift from container to press dye. This changes the height, diameter and mass of each sample from the expected. For microscopic imaging error is found in the lighting and pore determination. Since the pore size is dependent upon the lighting it makes it difficult in some samples to determine a pore and a dense area of the sample. This may lead to dense areas being classified as pores and vice versa. Figure 4 shows the trend of the expected, calculated and microscopic averages. Note that in all the volume ratios other than $1: 0.5$ the expected value is $3-14 \%$ greater than the calculated and microscopic averages.

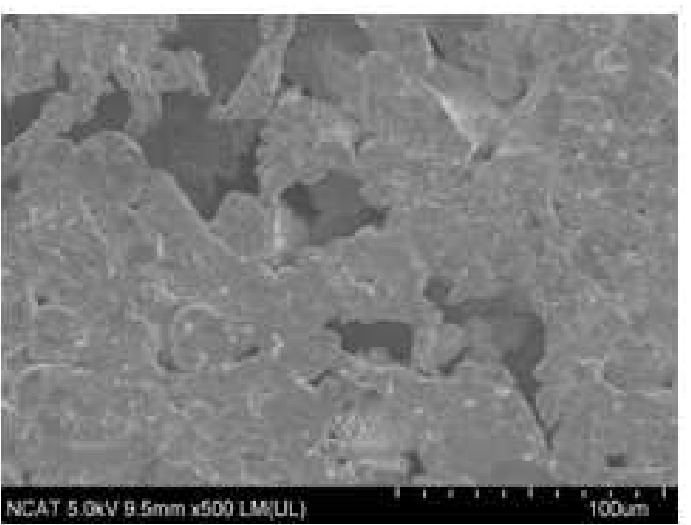

(b)

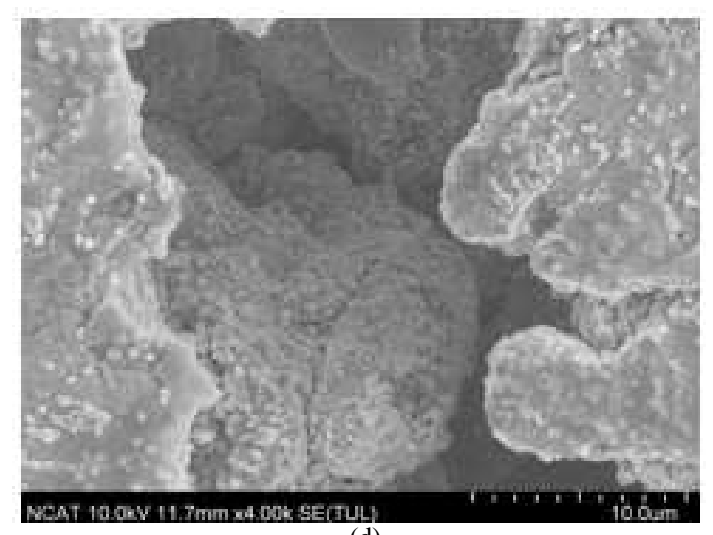

(d)

Fig. 2. (a) SEM image $50 \mathrm{x}$ (b) SEM image $500 \mathrm{x}$ (c) SEM image $2 \mathrm{Kx}$ (d) SEM image $5 \mathrm{Kx}$ 


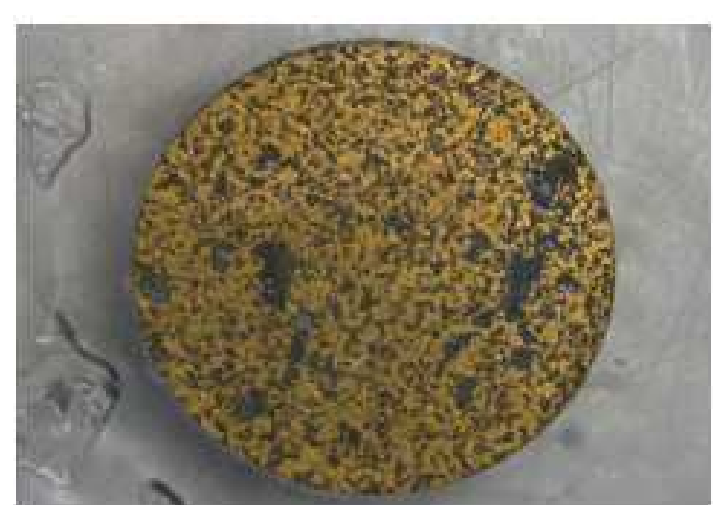

(a)

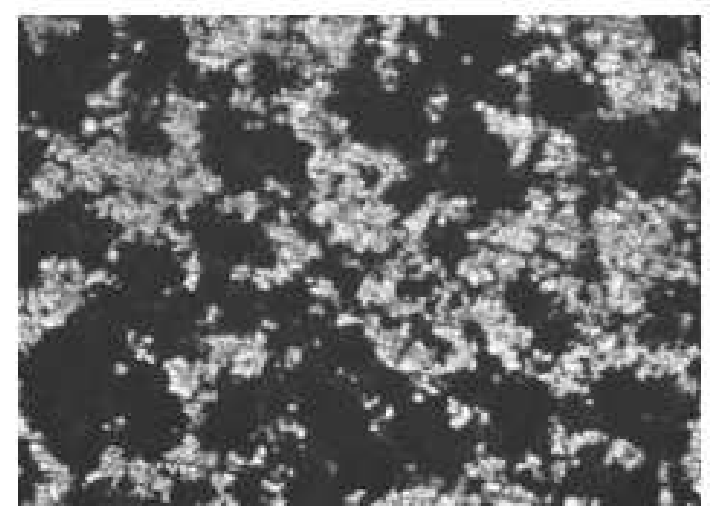

(c)

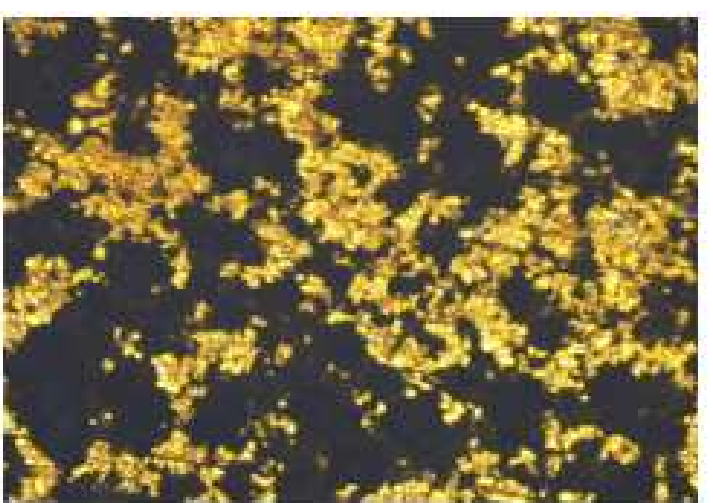

(b)

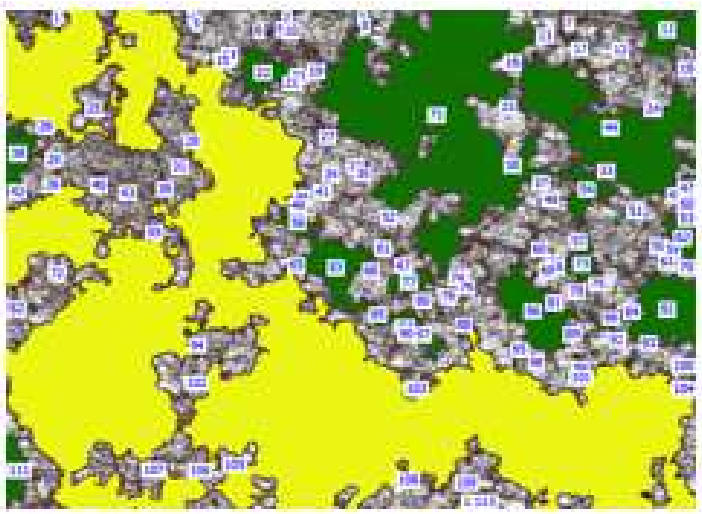

(d)

Fig. 3. (a) image of porous sample (b) microscopic image (c) black and white image (d) classification of pores

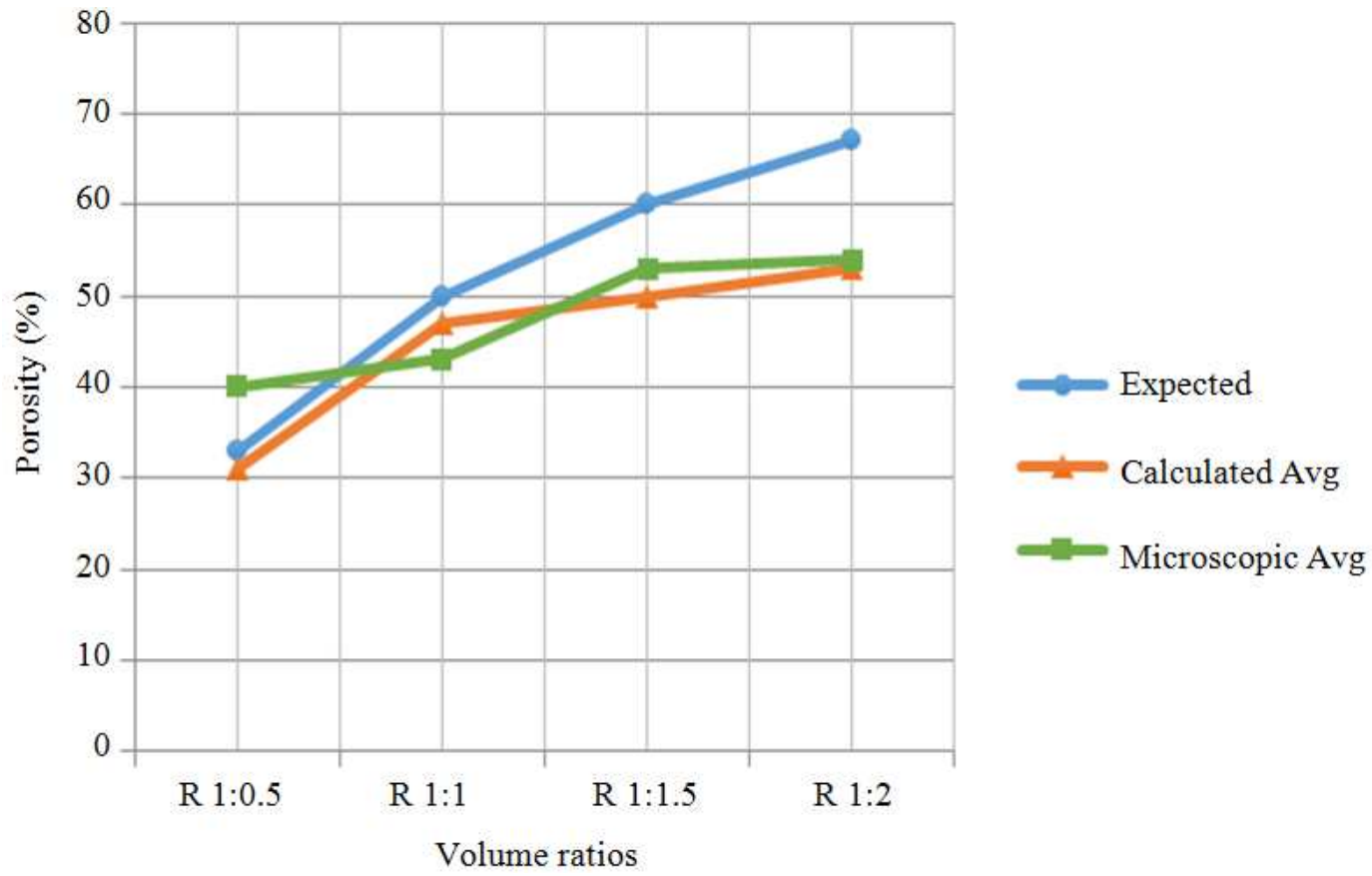

Fig. 4. Comparison of the expected, calculated and microscopic average 
Table 1. Comparison of the calculated porosity values to the expected values for each volume ratio, metal powder to space holder

\begin{tabular}{lllllll}
\hline Ratio & Sample 1 average & Sample 2 average & Sample 3 average & Sample 4 average & Grand average & Expected \\
\hline $1: 0.5$ & $31.98 \%$ & $32.01 \%$ & $37.08 \%$ & $24.51 \%$ & $31 \%$ & $33 \%$ \\
$1: 1$ & $47.51 \%$ & $51.91 \%$ & $42.35 \%$ & $47.59 \%$ & $47 \%$ & $50 \%$ \\
$1: 1.5$ & $49.81 \%$ & $49.08 \%$ & $49.08 \%$ & $53.21 \%$ & $50 \%$ & $60 \%$ \\
$1: 2$ & $53.39 \%$ & $54.10 \%$ & $51.99 \%$ & $52.46 \%$ & $53 \%$ & $67 \%$ \\
\hline
\end{tabular}

Table 2. Comparison of the microscopic porosity values to the expected values for each volume ratio, metal powder to space holder

\begin{tabular}{llllll}
\hline Ratio & Sample 1 average & Sample 2 average & Sample 3 average & Grand average & Expected \\
\hline $1: 0.5$ & $48.54 \%$ & $36.97 \%$ & $33.96 \%$ & $40 \%$ & $33 \%$ \\
$1: 1$ & $51.59 \%$ & $44.87 \%$ & $31.22 \%$ & $43 \%$ & $50 \%$ \\
$1: 1.5$ & $56.61 \%$ & $53.15 \%$ & $49.20 \%$ & $53 \%$ & $6 . \%$ \\
$1: 2$ & $58.21 \%$ & $63.52 \%$ & $40.50 \%$ & $54 \%$ & $67 \%$ \\
\hline
\end{tabular}

Table 3. Percent difference of the calculated and microscopic values to the expected values for each ratio

\begin{tabular}{llllll}
\hline Ration & Expected & Calculated & $\begin{array}{l}\text { Percent difference in } \\
\text { calculated Vs expected }\end{array}$ & Microscopic & $\begin{array}{l}\text { Percent difference in } \\
\text { macroscopic Vs expected }\end{array}$ \\
\hline $1: 0.5$ & $33 \%$ & $31 \%$ & $6 \%$ & $40 \%$ & $21 \%$ \\
$1: 1$ & $50 \%$ & $47 \%$ & $6 \%$ & $43 \%$ & $14 \%$ \\
$1: 1.5$ & $60 \%$ & $50 \%$ & $16 \%$ & $53 \%$ & $12 \%$ \\
$1: 2$ & $67 \%$ & $53 \%$ & $21 \%$ & $54 \%$ & $19 \%$ \\
\hline
\end{tabular}

\section{Conclusion}

The processing of porous brass samples by powder metallurgy using sodium chloride served as a viable construct for the study of porosity measurement methods. Sodium chloride has unique properties, which made it useful for a space holder material. A homogenous distribution of pores was a challenge to create. The process used to create the porous metals does not specify how to create homogenous pores. Trying to achieve a homogenous distribution for every sample was trial and error. Based upon the author's findings the calculation method is the most accurate method when determining porosity in a porous sample. Even though there was a margin of error in the calculated volume ratios the margin of error is greater in the microscopic imaging. As shown in Figure 4, the calculated average has the closest line of trend to the expected. In Table 3, the sum of the percent difference in calculated versus expected is lower than that of the microscopic. In the future, Archimedes' principle will be used to validate the calculated method as being the best method of choice when trying to determine the porosity in a sample. The main conclusions of the experimental work should be presented. The contribution of the work to the scientific community and its economic implications should be emphasized.

\section{Acknowledgement}

The North Carolina Louis Stokes Alliance for Minority Participation (NC-LSAMP) and Advanced Metallic Materials and Porous Structures Lab (AMMPS) sponsored this research. The authors would like to acknowledge the research advisor Dr. Cynthia Waters of North Carolina Agriculture and Technical State University. The authors would like to also acknowledge Dr. Boyce Collins of North Carolina Agriculture and Technical State University. The contents of this paper reflect the view of the authors, who are responsible for the facts and the accuracy of the data presented. This paper does not constitute a standard, specification, or regulation.

\section{Author's Contributions}

Ryan Yeargin: Participated in all experimental testing, data-analysis and contributed to the writing of the manuscript.

Rob Ramey: Participated in experimental plan and testing, data-analysis and contributed to the writing of the manuscript.

Cindy Waters: Participated in experimental plan, data-analysis and contributed to the writing of the manuscript.

\section{Ethics}

All rights reserved. No part of this publication may be reproduced or transmitted in any form or by any means, electronic or mechanical, including photocopy, or any information storage and retrieval system, without permission in writing from the publisher or authors.

\section{References}

Arifvianto, B. and J. Zhou, 2014. Fabrication of metallic biomedical scaffolds with the space holder method: A review. Materials, 7: 3588-3622.

DOI: $10.3390 / \mathrm{ma} 7053588$ 
ASI, 2015. Benefits of powder metal products manufacturing. Allied Sinterings, Inc.

Dictionary.com, 2015. Porosity. Dictionary.com Unabridged. Random House, Inc.

EMPA, 2015. What is powder metallurgy? European Powder Metallurgy Association.

Esen, Z. and S. Bor, 2011. Characterization of Ti-6Al$4 \mathrm{~V}$ alloy foams synthesized by space holder technique. Mater. Sci. Eng. A, 528: 3200-3209. DOI: $10.1016 /$ j.msea.2011.01.008

Hong, T.F., Z.X. Guo and R. Yang, 2008. Fabrication of porous titanium scaffold materials by a fugitive filler method. J. Mater. Sci. Mater. Med., 19: 3489-3495. DOI: 10.1007/s10856-008-3527-x

Matassi, F., A. Botti, L. Sirleo, C. Carulli and M. Innocenti, 2013. Porous metal for orthopedics implants. Clin. Cases Mineral Bone Metabolism, 10: 111-115. DOI: $10.11138 / \mathrm{ccmbm} / 2013.10 .2 .111$
Nakajima, H., 2010. Fabrication, properties and applications of porous metals with directional pores. Phys. Biol. Sci., 86: 884-899.

DOI: $10.2183 /$ pjab.86.884

Sharma, M., G.K. Gupta, O.P. Modi, B.K. Prasad and A.K. Gupta, 2011. Titanium foam through powder metallurgy route using acicular urea particles as space holder. Mater. Lett., 65: 3199-3201. DOI: 10.1016/j.matlet.2011.07.004

Tuncer, N. and G. Arslan, 2009. Designing compressive properties of titanium foams. J. Mater. Sci., 44: 1477-1484. DOI: 10.1007/s10853-008-3167-z 